\title{
A novel strategy for the synthesis of $6 H$-chromeno [4, 3-b] quinoline by intramolecular Heck cyclization
}

\author{
Morteza Shiri $^{\mathrm{a}, *}$, Mina Fathollahi-Lahroud ${ }^{\mathrm{a}}$ and Zahra Yasaei ${ }^{\mathrm{a}}$ \\ ${ }^{a}$ Department of Chemistry, Faculty of Physics and Chemistry, Alzahra University, Vanak, Tehran 1993893973, Iran, E-mail: mshiri@alzahra.ac.ir
}

\section{ARTICLE INFO}

Article history:

Received

Received in revised form

Accepted

Available online

Keywords:

Intramolecular Heck Reaction;

6H-chromeno [4,3-b] quinolines;

Palladium;

Indole.

\section{ABSTRACT}

A novel procedure for the synthesis of various derivatives of $6 H$-chromeno [4, 3-b] quinolines from intramolecular Heck reaction of 2-chloro-3-(phenoxymethyl) quinolines is described in this study. Interamolecular cyclisation of $N$-alkylated indoles was efficiently investigated as well. The reaction is catalyzed by bis (triphenylphosphine) palladium (II) dichloride in acetonitrile at $80{ }^{\circ} \mathrm{C}$.

2009 Elsevier Ltd. All rights reserved.

\section{Introduction}

An understanding of the details of $\mathrm{C}-\mathrm{C}$ bond formation of organometallic reactions, has persuaded chemists to use this strong key step in pharmaceutical and total synthesis of natural products. The precise choice of Pd catalyst and base will give arylation and vinylation of alkenes in a Heck procedure. ${ }^{1}$ Intramolecular Heck reaction is a straightforward approach to the synthesis of pharmaceuticals ${ }^{2}$ heterocycles and in total synthesis ${ }^{3}$, because very sterically hindered $\mathrm{C}-\mathrm{C}$ bond forming in mild conditions is possible. ${ }^{4}$ To the best of our knowledge, aryl chlorides are not generally very reactive reagents in Heck coupling reactions; however, by installing electron-accepting substitutions they become useful counterparts. ${ }^{5}$ It should be noted that Pd catalyzed intramolecular $\mathrm{C}-\mathrm{H}$ arylation in order to obtain fused dibenzo; 1, 6-naphthyridines has recently been reported. ${ }^{6}$

In accordance with the importance of activation of C-X bonds by intramolecular Heck procedure, quinolines and heteroaromatic compounds contain the moiety of significant frameworks, and they are a part of biological synthetic drugs or natural products. ${ }^{7}$ For example, three of the promising drugs of antimalarial depend on quinolines derivatives. ${ }^{8}$ Moreover, there are several reports of $6 H$-chromeno [4, 3-b] quinolines as estrogen receptor betaselective ligands ${ }^{9}$, anti-inflammatory and Ulcerogenic activities. ${ }^{10}$

Recently, 6H-chromeno [4, 3-b] quinolines 2 skeleton have been synthesized by different research groups. Among them ${ }^{11}$ are aza-Diels-Alder intramolecular $\mathrm{Cu}$-catalyzed reaction ${ }^{12}$ and copper-catalyzed reaction of arylpropynyloxy-benzonitriles with diaryliodonium triflates as arylating agent ${ }^{13}$ (Scheme 1). In continuation of our interest in chemistry of structures with quinoline core, ${ }^{14}$ we designed to prepare $6 H$-chromeno [4, 3-b] quinoline by the activation of $\mathrm{C}-\mathrm{Cl}$ bond of $\mathbf{1}$ in an intramolecular Heck coupling (Scheme 1).<smiles>[R2]c1ccc(/C=N/c2ccccc2)c(OCC#C)c1</smiles><smiles></smiles><smiles>[R]c1ccc2c(c1)-c1nc3ccccc3c([X])c1CO2</smiles><smiles>N#Cc1ccccc1OCC#Cc1ccccc1</smiles>

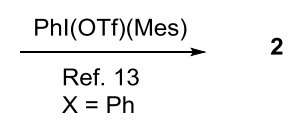<smiles>Clc1ccccc1</smiles><smiles>OCc1cc2ccccc2nc1Cl</smiles><smiles></smiles>

2

\section{Scheme 1}

\section{Results and discussion}

The research started by preparing the starting material of 2chloro-3- [(naphthalen-1-yloxy)methyl] quinoline 1a by the previous reported procedures. ${ }^{15}$ In this way, to a solution of $\mathbf{1 a}$ in acetonitrile, a palladium source of bis (triphenylphosphine) palladium (II) dichloride and $\mathrm{K}_{2} \mathrm{CO}_{3}$ as the base was added at 80 ${ }^{\circ} \mathrm{C}$. After $24 \mathrm{~h}$ the desired $6 \mathrm{H}$-chromeno [4, 3-b] quinoline 2a obtained the major product (Table 1 , entry 5 ). Then, the effect of various solvents, bases, ligands and catalysts in the reaction yield and time were explored (Table 1). By using acetone and toluene 
the yield was very low, but by using aprotic polar solvents such as DMF and DMA the product 2a was obtained in 70 and $85 \%$ yield respectively (Table 1, entries 1-4). By lowering the catalyst loading, the yield was decreased as well (Table 1, entries 5-8). Using other bases such as $t$-BuONa, $\mathrm{NaHCO}_{3}, \mathrm{NaOH}$ and $\mathrm{Cs}_{2} \mathrm{CO}_{3}$ have not noticeable effect on the yield of product. With $\mathrm{Et}_{3} \mathrm{~N}$ the reaction did not progress at all. Potassium carbonate was chosen as the best base. Next, various source of palladium catalyst such as $\mathrm{PdCl}_{2}, \mathrm{Pd}(\mathrm{OAc})_{2}, \mathrm{Pd}\left(\mathrm{PPh}_{3}\right)_{4}$ were checked and the yields were $40 \%, 80 \%$ and $0 \%$ respectively. The crucial role of palladium in the formation of the product when omitting the catalyst, even with strong bases of $\mathrm{KOH}, t-\mathrm{BuOK}$ and $\mathrm{Cs}_{2} \mathrm{CO}_{3}$ resulted without the desired product.

Table 1- Optimization of the reaction condition for the synthesis of $6 \mathrm{H}$-chromeno [4, 3-b] quinoline $\mathbf{2 a}$ from $1 \mathbf{a}^{\mathrm{a}}$

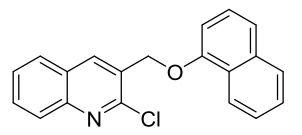

$1 \mathrm{a}$

\begin{tabular}{llllll}
\hline $\begin{array}{l}\text { Entr } \\
\mathrm{y}\end{array}$ & Solvent & Base & Catalyst $(10 \mathrm{~mol} \%)$ & Ligand & Yield(\%) \\
& & & & \\
\hline 1 & Acetone & $\mathrm{K}_{2} \mathrm{CO}_{3}$ & $\mathrm{PdCl}_{2}\left(\mathrm{PPh}_{3}\right)_{2}$ & - & 20
\end{tabular}

${ }^{1} \mathrm{H}-\mathrm{NMR}$ spectra of $\mathbf{2 a}$ and $\mathbf{2 b}$ have some interesting information about the 3D-properties of the synthesized molecules. The indicated proton in 2a (Fig. 1) which is deshielded aromatic proton, appeared as a doublet in chemical shift of 8.64 . While such a proton in $\mathbf{2} \mathbf{b}$ appeared as a doublet in $9.99 \mathrm{ppm}$. This is due to the proximity of the hydrogen to the electronegative nitrogen of quinoline ring as well as anisotropic effect.<smiles>c1ccc2nc3c(cc2c1)COc1ccc2ccccc2c1-3</smiles>

2b<smiles>[R6][R6]([H])([H])[C@H](C)c1cc2ccccc2c2c1-c1nc3ccccc3cc1CO2</smiles>

Figure 1.

Table 2. Synthesis of various $6 H$-chromeno [4,3-b] quinolines 2a-n

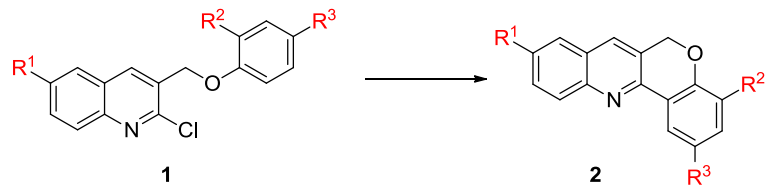

2 Toluene $\quad \mathrm{K}_{2} \mathrm{CO}_{3} \quad \mathrm{PdCl}_{2}\left(\mathrm{PPh}_{3}\right)_{2} \quad$ - 42

$3 \quad \mathrm{DMF} \quad \mathrm{K}_{2} \mathrm{CO}_{3} \quad \mathrm{PdCl}_{2}\left(\mathrm{PPh}_{3}\right)_{2} \quad$ - 70

$4 \quad \mathrm{DMA} \quad \mathrm{K}_{2} \mathrm{CO}_{3} \quad \mathrm{PdCl}_{2}\left(\mathrm{PPh}_{3}\right)_{2} \quad$ - $\quad 84$

$5 \quad \begin{array}{llllll}\mathrm{CH}_{3} \mathrm{CN} & \mathrm{K}_{2} \mathrm{CO}_{3} & \mathrm{PdCl}_{2}\left(\mathrm{PPh}_{3}\right)_{2} & - & 90\end{array}$

$6 \quad \mathrm{CH}_{3} \mathrm{CN} \quad \mathrm{K}_{2} \mathrm{CO}_{3} \quad \mathrm{PdCl}_{2}\left(\mathrm{PPh}_{3}\right)_{2}(8 \%) \quad-\quad 81$

$7 \quad \mathrm{CH}_{3} \mathrm{CN} \quad \mathrm{K}_{2} \mathrm{CO}_{3} \quad \mathrm{PdCl}_{2}\left(\mathrm{PPh}_{3}\right)_{2}(5 \%) \quad-\quad 43$

$8 \quad \mathrm{CH}_{3} \mathrm{CN} \quad \mathrm{K}_{2} \mathrm{CO}_{3} \quad \mathrm{PdCl}_{2}\left(\mathrm{PPh}_{3}\right)_{2}(2 \%) \quad-\quad$ Trace

$9 \quad \mathrm{CH}_{3} \mathrm{CN} \quad \mathrm{NaOAc} \quad \mathrm{PdCl}_{2}\left(\mathrm{PPh}_{3}\right)_{2} \quad-\quad$ N.R

${ }^{\mathrm{a}}$ 1a (1 mmol), Cat. (10 mol\%), Base (2 mmol), Solvent (5 mL), reflux, $24 \mathrm{~h}$

${ }^{\mathrm{b}}$ Isolated yields

The optimized reaction condition, $\mathrm{Pd}\left(\mathrm{PPh}_{3}\right)_{2} \mathrm{Cl}_{2}(10 \mathrm{~mol} \%)$ as catalyst, $\mathrm{K}_{2} \mathrm{CO}_{3}$ as the base, reflux of $\mathrm{CH}_{3} \mathrm{CN}$, reaction time of 24 $\mathrm{h}$, was examined on the generality of the reaction (Table 2).

As is clear from Table 2, electron-withdrawing and electrondonating groups on the both sides of ether $\mathbf{1}$ participated properly in the intramolecular cyclisation towards four and five membered azaheterocycles. Furthermore, tertiary butyl, as a good candidate of hindered groups, worked well to give $\mathbf{2 g}$ in $70 \%$ yield (Table 2).

Next, for further exploration of the scope of the reaction, 3[(1H-indol-1-yl) methyl]-2-chloroquinoline $\mathbf{3 a}$ and 2-chloro-3[(5-chloro-1H-indol-1-yl] methyl) quinoline $\mathbf{3 b}$ were prepared (Scheme 2). The synthesis of five cycle indolo quinolines 4a and 4b were also facilitated by this method as demonstrated in Scheme 2.

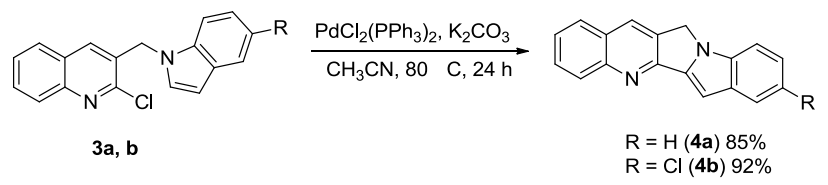

Scheme 2 


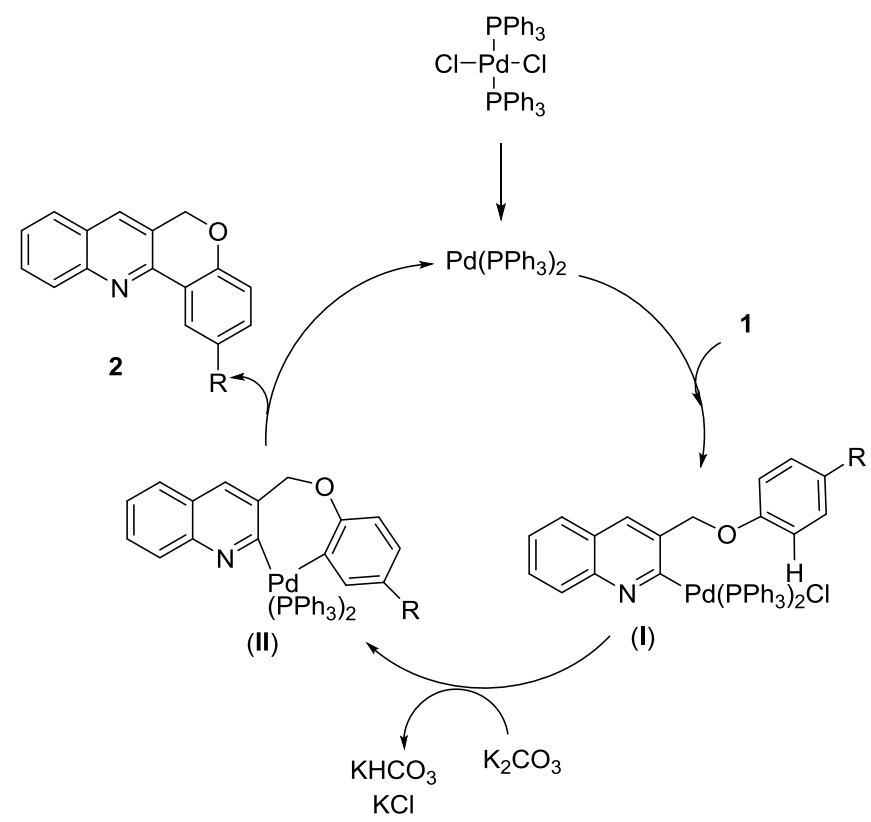

Scheme 3. The plausible mechanism of the reaction

\section{Conclusions}

In summary, a number of $6 H$-chromeno (4, 3-b) quinolines in a convenient and simple intramolecular cyclization were synthesized. 2-Chloro-3- (aryloxymethyl) quinolines were the starting materials which in front of 10 mol\% of a palladium catalyst and $\mathrm{K}_{2} \mathrm{CO}_{3}$ in acetonitrile afforded the desired scaffolds. Aryl chlorides are rare starting reagents in Heck coupling procedures because they are considered unreactive in comparison to aryl bromides and iodides. The conjugates of important quinoline pharmacophore with chromen ring would be interesting in terms of its biological effects. Moreover, the reaction has the advantage of tolerance of a wide range of functional groups.

\section{Experimental section}

\section{General}

The chemicals were purchased from Fluka, Merck and Aldrich chemical companies. Melting points are uncorrected. IR spectra were recorded on a Shimadzu Infra-Red Spectroscopy IR-435. Nuclear magnetic resonance (NMR) spectra were recorded on a Bruker Avance $400 \mathrm{MHz}$ Spectrometer in $\mathrm{CDCl}_{3}$ as a solvent. The elemental analysis was carried out with a Leco CHNS model 932. Mass spectra were recorded on Agilent Technology (HP) 5973 Network Mass Selective Detector operating at an ionization potential of $70 \mathrm{eV}$.

The typical procedure for the synthesis of $6 \mathrm{H}$-benzo $[7,8]$ chromeno [4, 3-b] quinolone (2a). To a stirring solution of 2-chloro-3-[(naphthalen-1-yloxy) methyl] quinoline 4a (1 $\mathrm{mmol})$ in acetonitrile $(5 \mathrm{~mL}), 10 \mathrm{~mol} \%$ of bis (triphenylphosphine) palladium (II) dichloride and potassium carbonate (2 $\mathrm{mmol})$ were added. The reaction stirred at reflux condition for $24 \mathrm{~h}$. The completion of the reaction was monitored by TLC. By filtration of the crude reaction and the evaporation of its solvent, the product was purified by a column chromatography (n-hexan: ethyl acetate 9:1).

\section{Supplementary Material}

6H-Benzo [7, 8]chromeno [4,3-b]quinoline (2a). Colorless needle crystal mp: $139-141{ }^{\circ} \mathrm{C}$ (from $\left.\mathrm{MeOH}\right) .{ }^{1} \mathrm{H}-\mathrm{NMR}(400$ $\left.\mathrm{MHz}, \mathrm{CDCl}_{3}\right): \delta=5.60(\mathrm{~s}, 2 \mathrm{H}), 7.51-7.60(\mathrm{~m}, 3 \mathrm{H}), 7.65$ (d, $J=$ $8.4 \mathrm{~Hz}, 1 \mathrm{H}), 7.73$ (t, $J=7.6 \mathrm{~Hz}, 1 \mathrm{H}), 7.84$ (d, $J=8.6 \mathrm{~Hz}, 1 \mathrm{H})$,
7.88 (d, $J=7.2 \mathrm{~Hz}, 1 \mathrm{H}), 7.94$ (s, 1H), 8.19 (d, $J=8.4 \mathrm{~Hz}, 1 \mathrm{H})$, 8.33 (d, $J=7.0 \mathrm{~Hz}, 1 \mathrm{H}), 8.59$ (d, $J=8.4 \mathrm{~Hz}, 1 \mathrm{H}) \mathrm{ppm}{ }^{13} \mathrm{C}-\mathrm{NMR}$ $\left(100 \mathrm{MHz}, \mathrm{CDCl}_{3}\right): \delta=68.9,117.6,121.9,122.1,122.4,124.8$, $125.1,125.8,126.1,127.4,127.5,127.6,127.8,129.4,129.5$, 130.7, 135.8, 148.5, 149.5, 153.9 ppm. Mass: $\mathrm{m} / \mathrm{z} 283(\mathrm{M}+)$ (calcd for $\mathrm{C}_{20} \mathrm{H}_{13} \mathrm{NO}$ : 283.32). FT-IR (KBr): $v_{\max }: 1100$ (m), 1260 (m), 1571 (s), 2851 (m), 3061 (s) $\mathrm{cm}^{-1}$. Analytically calculated for $\mathrm{C}_{20} \mathrm{H}_{13} \mathrm{NO}$ : C 84.78, H 4.62, N, 4.94\%. Found: C 84.55; H 4.51; N 4.72\%.

8H-Benzo [5, 6]chromeno [4,3-b]quinoline (2b). Colorless needle crystal, mp: $127-130{ }^{\circ} \mathrm{C}$ (from MeOH). ${ }^{1} \mathrm{H}-\mathrm{NMR}(400$ $\left.\mathrm{MHz}, \mathrm{CDCl}_{3}\right): \delta=5.36(\mathrm{~s}, 2 \mathrm{H}), 7.27(\mathrm{~d}, J=8.8 \mathrm{~Hz}, 1 \mathrm{H}), 7.51(\mathrm{t}$, $J=7.6 \mathrm{~Hz}, 1 \mathrm{H}), 7.56(\mathrm{t}, J=8.0 \mathrm{~Hz}, 1 \mathrm{H}), 7.70-7.78(\mathrm{~m}, 2 \mathrm{H})$, $7.84-7.91$ (m, 3H ), 8.01 (s, 1H), 8.26 (d, $J=8.4 \mathrm{~Hz}, 1 \mathrm{H}), 9.99$ $(\mathrm{d}, J=8.8 \mathrm{~Hz}, 1 \mathrm{H}) \mathrm{ppm} .{ }^{13} \mathrm{C}-\mathrm{NMR}\left(100 \mathrm{MHz} \mathrm{CDCl}_{3}\right): \delta=68.9$, $116.2,118.3,121.6,124.5,126.3,126.7,127.1,127.2,127.9$, 128.4, 129.4, 129.6, 130.7, 130.8, 131.3, 133.0, 148.0, 150.8, 157.8 ppm. Mass: $m / z 283\left(\mathrm{M}+\right.$ ) (calcd for $\mathrm{C}_{20} \mathrm{H}_{13} \mathrm{NO}$ : 283.32).FT-IR (KBr): $v_{\max }$ : 1100 (s), 1260 (s), 1742 (m), 2962 (w) $\mathrm{cm}^{-1}$. Analytically calculated for $\mathrm{C}_{20} \mathrm{H}_{13} \mathrm{NO}$ : C 84.78, H 4.62, N, 4.94\%. Found: C 84.61; H 4.33; N 4.79\%.

6H-Chromeno [4, 3-b] quinoline (2c). Colorless needle crystal, mp: 85-89 ${ }^{\circ} \mathrm{C}$ (from $\mathrm{MeOH}$ ). ${ }^{1} \mathrm{H}-\mathrm{NMR}$ (400 MHz, $\left.\mathrm{CDCl}_{3}\right): \delta=5.39(\mathrm{~s}, 2 \mathrm{H}), 7.06(\mathrm{~d}, J=8.4 \mathrm{~Hz}, 1 \mathrm{H}), 7.21(\mathrm{t}, J=8.0$ $\mathrm{Hz}, 1 \mathrm{H}), 7.41(\mathrm{t}, J=8.0 \mathrm{~Hz}, 1 \mathrm{H}), 7.52(\mathrm{t}, J=7.6 \mathrm{~Hz}, 1 \mathrm{H}), 7.73(\mathrm{t}$, $J=7.6 \mathrm{~Hz}, 1 \mathrm{H}), 7.80$ (d, $J=8.4 \mathrm{~Hz}, 1 \mathrm{H}), 7.89$ (s, $1 \mathrm{H}), 8.17$ (d, $J$ $=8.4 \mathrm{~Hz}, 1 \mathrm{H}), 8.53$ (dd $J=7.6 \mathrm{~Hz}, J=1.6 \mathrm{~Hz}, 1 \mathrm{H}) \mathrm{ppm} .{ }^{13} \mathrm{C}-$ NMR (100 MHz, $\left.\mathrm{CDCl}_{3}\right): \delta=68.4,117.3,122.6,123.2,125.2$, 125.6, 126.3, 127.4, 127.6, 129.4, 129.5, 130.9, 131.9, 148.3, 149.3, 157.4 ppm. Mass: $\mathrm{m} / \mathrm{z} 232$ (M-1) (calcd for $\mathrm{C}_{16} \mathrm{H}_{11} \mathrm{NO}$ : 233.28). FT-IR (KBr): $v_{\max }$ : 1017 (s), 1238 (m), 1605 (m), 2900 (w) $\mathrm{cm}^{-1}$. Analytically calculated for $\mathrm{C}_{16} \mathrm{H}_{11} \mathrm{NO}$ : C 82.38, H 4.75, N, 6.00\%. Found: C 82.19; H 4.59; N 6.12\%.

2-Chloro-6H-chromeno [4, 3-b] quinoline (2d). Yellow needle crystal, mp: $113-117{ }^{\circ} \mathrm{C}$ (from MeOH). ${ }^{1} \mathrm{H}-\mathrm{NMR}(400$ $\left.\mathrm{MHz}, \mathrm{CDCl}_{3}\right): \delta=5.38(\mathrm{~d}, J=1.2 \mathrm{~Hz}, 2 \mathrm{H}), 6.98(\mathrm{~d}, J=8.8 \mathrm{~Hz}$, $1 \mathrm{H}), 7.33$ (dd, $J=8.8 \mathrm{~Hz}, J=2.8 \mathrm{~Hz}, 1 \mathrm{H}), 7.55$ (t, $J=8.8 \mathrm{~Hz}$, $1 \mathrm{H}), 7.74$ (t, $J=8.0 \mathrm{~Hz}, 1 \mathrm{H}), 7.80$ (d, $J=8.4 \mathrm{~Hz}, 1 \mathrm{H}$ ), 7.91 (s, $1 \mathrm{H}), 8.17$ (d, $J=8.4 \mathrm{~Hz}, 1 \mathrm{H}), 8.49$ (d, $J=2.8 \mathrm{~Hz}, 1 \mathrm{H}) \mathrm{ppm} .{ }^{13} \mathrm{C}-$ NMR (100 MHz, $\left.\mathrm{CDCl}_{3}\right): \delta=68.3,118.6,120.9,124.8,125.5$, 126.9, 127.5, 127.8, 129.4, 129.6, 132.2, 133.7, 141.2, 151.0, 151.0, 155.9 ppm. Mass: $\mathrm{m} / \mathrm{z} 266$ (M-1) (calcd for $\mathrm{C}_{16} \mathrm{H}_{10} \mathrm{ClNO}$ : 267.05). FT-IR (KBr): $v_{\max }: 736$ (m), 1081 (m), 1242 (m), 3044 (w) $\mathrm{cm}^{-1}$. Analytically calculated for $\mathrm{C}_{16} \mathrm{H}_{10}$ ClNO: $\mathrm{C} 71.78, \mathrm{H}$ 3.77, N, 5.23\%. Found: C 71.53; H 3.81; N 5.19\%.

2-Fluoro-6H-chromeno [4, 3-b] quinoline (2e). Yellow needle crystal, mp: 97-100 ${ }^{\circ} \mathrm{C}$ (from $\mathrm{MeOH}$ ). ${ }^{1} \mathrm{H}-\mathrm{NMR}(400 \mathrm{MHz}$, $\left.\mathrm{CDCl}_{3}\right): \delta=5.37(\mathrm{~d}, J=0.8 \mathrm{~Hz}, 2 \mathrm{H}), 6.99-7.03(\mathrm{~m}, 1 \mathrm{H}), 7.09$ (dd, $J=8.0 \mathrm{~Hz}, J=3.2 \mathrm{~Hz}, 1 \mathrm{H}), 7.55(\mathrm{t}, J=6.8 \mathrm{~Hz}, 1 \mathrm{H}), 7.74(\mathrm{t}$, $J=6.4 \mathrm{~Hz}, 1 \mathrm{H}), 7.81$ (d, $J=8.4 \mathrm{~Hz}, 1 \mathrm{H}), 7.92$ (s, 1H), 7.16 (d, $J$ $=9.2 \mathrm{~Hz}, 1 \mathrm{H}), 8.19$ (dd, $J=9.2 \mathrm{~Hz}, J=3.2 \mathrm{~Hz}, 1 \mathrm{H}) \mathrm{ppm} .{ }^{13} \mathrm{C}-$ NMR (100 MHz, $\left.\mathrm{CDCl}_{3}\right): \delta=68.5,118.5,118.6,122.2,125.0$, 126.7, 127.1, 127.7, 129.5, 129.8, 131.1, 148.2, 153.4, 157.2, 159.6 ppm. Mass: $\mathrm{m} / \mathrm{z} 251\left(\mathrm{M}^{+}\right)$(calcd for $\mathrm{C}_{16} \mathrm{H}_{10} \mathrm{FNO}$ : 251.07). FT-IR (KBr): $v_{\max }: 806(\mathrm{~m}), 1113(\mathrm{~m}), 1188(\mathrm{~m}), 3046(\mathrm{w}) \mathrm{cm}^{-1}$. Analytically calculated for $\mathrm{C}_{16} \mathrm{H}_{10}$ FNO: C 76.48, H 4.01, F 7.56 N, 5.57\%. Found: C 76.18; H 4.18; N 5.31\%.

6H-Chromeno [4, 3-b] quinoline-2-carbonitrile (2f). Yellow needle crystal, mp: $137-140{ }^{\circ} \mathrm{C}$ (from MeOH). ${ }^{1} \mathrm{H}-\mathrm{NMR}(400$ $\left.\mathrm{MHz}, \mathrm{CDCl}_{3}\right): \delta=5.49(\mathrm{~d}, J=0.8 \mathrm{~Hz}, 2 \mathrm{H}), 7.10(\mathrm{~d}, J=8.4 \mathrm{~Hz}$, $1 \mathrm{H}), 7.58(\mathrm{t}, J=6.8 \mathrm{~Hz}, 1 \mathrm{H}), 7.64(\mathrm{dd}, J=8.6 \mathrm{~Hz}, J=2.4 \mathrm{~Hz}$, $1 \mathrm{H}), 7.77$ (t, $J=5.6 \mathrm{~Hz}, 1 \mathrm{H}), 7.82(\mathrm{~d}, \mathrm{~J}=8.0 \mathrm{~Hz}, 1 \mathrm{H}$ ), 7.93 (s, $1 \mathrm{H}), 8.17$ (d, $J=8.4 \mathrm{~Hz}, 1 \mathrm{H}), 8.86(\mathrm{~d}, J=2.0 \mathrm{~Hz}, 1 \mathrm{H}) \mathrm{ppm} .{ }^{13} \mathrm{C}-$ 
NMR $\left(100 \mathrm{MHz}, \mathrm{CDCl}_{3}\right): \delta=68.6,106.0,118.6,118.9,123.9$, $127.1,127.5,127.9,129.6,130.1,130.2,131.4,134.9,146.5$, 148.3, 160.2, 175.1 ppm. Mass: $\mathrm{m} / \mathrm{z} 258\left(\mathrm{M}^{+}\right.$) (calcd for $\mathrm{C}_{17} \mathrm{H}_{10} \mathrm{~N}_{2} \mathrm{O}:$ 258.08). FT-IR (KBr): $v_{\max }: 1130$ (s), 1235 (s), 2223 (s), $3068(\mathrm{w}) \mathrm{cm}^{-1}$. Analytically calculated for $\mathrm{C}_{17} \mathrm{H}_{10} \mathrm{~N}_{2} \mathrm{O}: \mathrm{C}$ 79.06, H 3.90, N, 10.85\%. Found: C 78.88; H 3.71; N 10.77\%.

2-(tert-Butyl)-6H-chromeno [4, 3-b] quinoline (2g). Yellow needle crystal, mp: $68-71{ }^{\circ} \mathrm{C}$ (from MeOH). ${ }^{1} \mathrm{H}-\mathrm{NMR}(400 \mathrm{MHz}$, $\left.\mathrm{CDCl}_{3}\right): \delta=1.46(\mathrm{~s}, 9 \mathrm{H}), 5.36(\mathrm{~d}, J=0.8 \mathrm{~Hz}, 2 \mathrm{H}), 7.00(\mathrm{~d}, J=$ $8.8 \mathrm{~Hz}, 1 \mathrm{H}), 7.46(\mathrm{dd}, J=8.6 \mathrm{~Hz}, J=2.8 \mathrm{~Hz}, 1 \mathrm{H}), 7.52(\mathrm{t}, J=7.6$ $\mathrm{Hz}, 1 \mathrm{H}), 7.72$ (t, $J=7.6 \mathrm{~Hz}, 1 \mathrm{H}), 7.80$ (d, $J=8.0 \mathrm{~Hz}, 1 \mathrm{H}$ ), 7.89 (s, 1H), 8.19 (d, $J=8.8 \mathrm{~Hz}, 1 \mathrm{H}), 8.55$ (d, $J=2.4 \mathrm{~Hz}, 1 \mathrm{H}) \mathrm{ppm}$. ${ }^{13} \mathrm{C}-\mathrm{NMR}\left(100 \mathrm{MHz}, \mathrm{CDCl}_{3}\right): \delta=31.5,34.6,68.4,114.4,116.9$, $121.4,121.9,125.5,126.2,126.5,127.3,127.4,127.5,129.4$, 133.3, 136.7, 141.2, 155.4 ppm. Mass: $\mathrm{m} / \mathrm{z} 289\left(\mathrm{M}^{+}\right)$(calcd for $\mathrm{C}_{20} \mathrm{H}_{19} \mathrm{NO}$ : 289.15). Analytically calculated for $\mathrm{C}_{20} \mathrm{H}_{19} \mathrm{NO}$ : C 83.01, H 6.62, N, 4.84\%. Found: C 83.15; H 6.77; N 4.77\%.

4-Methyl-6H-chromeno [4, 3-b] quinoline (2h). Yellow needle crystal, mp: 102-105 ${ }^{\circ} \mathrm{C}$ (from $\left.\mathrm{MeOH}\right) .{ }^{1} \mathrm{H}-\mathrm{NMR}(400$ $\left.\mathrm{MHz}, \mathrm{CDCl}_{3}\right): \delta=2.34(\mathrm{~s}, 3 \mathrm{H}), 5.40(\mathrm{~d}, J=0.8 \mathrm{~Hz}, 2 \mathrm{H}), 7.10(\mathrm{t}$, $J=7.6 \mathrm{~Hz}, 1 \mathrm{H}$ ), 7.27 (d, $J=7.0 \mathrm{~Hz}, 2 \mathrm{H}), 7.52$ (t, $J=7.4 \mathrm{~Hz}$, $1 \mathrm{H}), 7.72$ (t, $J=7.8 \mathrm{~Hz}, 1 \mathrm{H}), 7.80$ (d, $J=7.4 \mathrm{~Hz}, 1 \mathrm{H}), 7.91$ (s, $1 \mathrm{H}), 8.16(\mathrm{~d}, J=8.4 \mathrm{~Hz}, 1 \mathrm{H}), 8.37$ (d, $J=8.0 \mathrm{~Hz}, 1 \mathrm{H}) \mathrm{ppm} .{ }^{13} \mathrm{C}-$ NMR $\left(100 \mathrm{MHz}, \mathrm{CDCl}_{3}\right): \delta=15.8,68.3,121.9,122.8,123.2$, 125.3 , 126.2, 126.6, 127.4, 127.5, 129.4, 130.7, 133.1, 148.4, 149.5, 155.6 ppm. Mass: $\mathrm{m} / \mathrm{z} 246$ (M-1) (calcd for $\mathrm{C}_{17} \mathrm{H}_{13} \mathrm{NO}$ : 247.29). FT-IR (KBr): $v_{\max }$ : 744 (s), 1019 (m), 1204 (s), 1462 (s), 3049 (w) $\mathrm{cm}^{-1}$. Analytically calculated for $\mathrm{C}_{17} \mathrm{H}_{13} \mathrm{NO}$ : C 82.57, $\mathrm{H}$ 5.30, N, 5.66\%. Found: C 82.41; H 5.17; N 5.51\%.

9-Bromo-6H-chromeno [4, 3-b] quinoline (2i). Yellow needle crystal, mp: $118-120{ }^{\circ} \mathrm{C}$ (from $\mathrm{MeOH}$ ). ${ }^{1} \mathrm{H}-\mathrm{NMR}(400 \mathrm{MHz}$, $\left.\mathrm{CDCl}_{3}\right): \delta=5.38(\mathrm{~d}, J=0.8 \mathrm{~Hz}, 2 \mathrm{H}), 6.77(\mathrm{~d}, J=8.8 \mathrm{~Hz}, 1 \mathrm{H})$, 7.00 (d, $J=8.8 \mathrm{~Hz}, 1 \mathrm{H}), 7.67(\mathrm{~m}, 1 \mathrm{H}), 7.80(\mathrm{~m}, 1 \mathrm{H}), 7.83$ (s, $1 \mathrm{H}), 8.01(\mathrm{~d}, J=9.2 \mathrm{~Hz}, 1 \mathrm{H}), 8.09(\mathrm{~d}, J=8.8 \mathrm{~Hz}, 1 \mathrm{H}), 8.46(\mathrm{~d}, J$ $=2.8 \mathrm{~Hz}, 1 \mathrm{H}) \mathrm{ppm} .{ }^{13} \mathrm{C}-\mathrm{NMR}\left(100 \mathrm{MHz}, \mathrm{CDCl}_{3}\right): \delta=68.3$, 116.4, 118.9, 121.1, 124.0, 125.1, 126.1, 127.9, 130.1, 130.7, 131.1, 131.4, 131.8, 133.4, 146.7, 155.8 ppm. FT-IR (KBr): $v_{\max }$ : $650(\mathrm{w}), 1130$ (w), 1230 (m), 3020 (w) cm $\mathrm{cm}^{-1}$. Analytically calculated for $\mathrm{C}_{16} \mathrm{H}_{10} \mathrm{BrNO}$ : C 61.56, H 3.23, N, 4.49\%. Found: C 61.27; H 3.41; N 4.72\%.

9-Chloro-6H-chromeno [4, 3-b] quinoline-2-carbonitrile (2j). Yellow needle crystal, mp: $137-140{ }^{\circ} \mathrm{C}$ (from MeOH). ${ }^{1} \mathrm{H}-\mathrm{NMR}$ $\left(400 \mathrm{MHz}, \mathrm{CDCl}_{3}\right): \delta=5.49(\mathrm{~d}, J=0.8 \mathrm{~Hz}, 2 \mathrm{H}), 7.11(\mathrm{~d}, J=8.4$ $\mathrm{Hz}, 1 \mathrm{H}$ ), 7.65 (dd, $J=8.4 \mathrm{~Hz}, J=2.0 \mathrm{~Hz}, 1 \mathrm{H}$ ), 7.70 (dd, $J=9.2$ $\mathrm{Hz}, J=2.4 \mathrm{~Hz}, 1 \mathrm{H}), 7.81$ (d, $J=2.4 \mathrm{~Hz}, 1 \mathrm{H}), 7.85$ (s, 1H), 8.10 (d, $J=8.8 \mathrm{~Hz}, 1 \mathrm{H}), 8.82$ (d, $J=2.0 \mathrm{~Hz}, 1 \mathrm{H}) \mathrm{ppm} .{ }^{13} \mathrm{C}-\mathrm{NMR}(100$ $\left.\mathrm{MHz} \mathrm{CDCl}_{3}\right): \delta=68.4,106.2,118.7,118.8,123.5,124.9,126.2$, 128.4, 130.3, 130.4, 131.1, 131.2, 132.8, 135.2, 146.7, 146.8, 160.2 ppm. Mass: $\mathrm{m} / \mathrm{z} 291$ (M-1) (calcd for $\mathrm{C}_{17} \mathrm{H}_{9} \mathrm{ClN}_{2} \mathrm{O}$ : 292.04). FT-IR (KBr): $v_{\max }: 779$ (m), 1130 (m), 1235 (s), 3067 (w) $\mathrm{cm}^{-1}$. Analytically calculated for $\mathrm{C}_{17} \mathrm{H}_{9} \mathrm{ClN}_{2} \mathrm{O}: \mathrm{C}$ 69.75, $\mathrm{H}$ 3.10, N, 9.57\%. Found: C 69.58; H 3.17; N 9.71\%.

9-Methyl-6H-chromeno [4, 3-b] quinoline-2-carbonitrile (2k) Colorless needle crystal, mp: $147-150{ }^{\circ} \mathrm{C}$ (from MeOH). ${ }^{1} \mathrm{H}-$ NMR $\left(400 \mathrm{MHz}, \mathrm{CDCl}_{3}\right): \delta=2.58(\mathrm{~s}, 3 \mathrm{H}), 5.47(\mathrm{~d}, J=0.8 \mathrm{~Hz}$, 2H), 7.09 (d, $J=8.4 \mathrm{~Hz}, 1 \mathrm{H}), 7.58-7.64(\mathrm{~m}, 3 \mathrm{H}), 7.83(\mathrm{~s}, 1 \mathrm{H})$, 8.05 (d, $J=8.4 \mathrm{~Hz}, 1 \mathrm{H}), 8.83(\mathrm{~d}, J=2.0 \mathrm{~Hz}, 1 \mathrm{H}) \mathrm{ppm} .{ }^{13} \mathrm{C}-\mathrm{NMR}$ $\left(100 \mathrm{MHz}, \mathrm{CDCl}_{3}\right): \delta=21.6,68.6,106.0,118.5,118.9,123.9$, $124.0,126.4,127.9,129.2,130.1,130.7,132.4,134.7,137.2$, 145.7, 146.9, 160.1 ppm. Mass: $\mathrm{m} / \mathrm{z} 271$ (M-1) (calcd for $\mathrm{C}_{18} \mathrm{H}_{12} \mathrm{~N}_{2} \mathrm{O}:$ 272.09). FT-IR (KBr): $v_{\max }: 1122$ (m), 1236 (s), 2233 (s), $2855(\mathrm{w}), 3030(\mathrm{w}) \mathrm{cm}^{-1}$. Analytically calculated for
$\mathrm{C}_{18} \mathrm{H}_{12} \mathrm{~N}_{2} \mathrm{O}$ : C 79.39, $\mathrm{H}$ 4.44, N, 10.29\%. Found: C 79.47; $\mathrm{H}$ 4.62; N 10.51\%.

2-Fluoro-9-methyl-6H-chromeno [4, 3-b] quinoline (2l). Yellow needle crystal, mp: 92-95 ${ }^{\circ} \mathrm{C}$ (from MeOH). ${ }^{1} \mathrm{H}-\mathrm{NMR}$ $\left(400 \mathrm{MHz}, \mathrm{CDCl}_{3}\right): \delta=2.57(\mathrm{~s}, 3 \mathrm{H}), 5.36(\mathrm{~d}, J=0.8 \mathrm{~Hz}, 2 \mathrm{H})$, 7.00 (m, 1H), 7.08 (m, 1H), 7.54-7.58 (m, 2H), 7.83 (s, 1H), 8.06 (d, $J=9.2 \mathrm{~Hz}, 1 \mathrm{H}$ ), 8.18 (dd, $J=8.8 \mathrm{~Hz}, J=3.2 \mathrm{~Hz}, 1 \mathrm{H}$ ) ppm. ${ }^{13} \mathrm{C}-\mathrm{NMR}\left(100 \mathrm{MHz}, \mathrm{CDCl}_{3}\right): \delta=21.6,68.5,111.3,1115,118.4$, 118.5, 118.6, 125.0, 126.3, 127.8, 128.9, 130.6, 132.2, 157.2, 159.6 ppm. Mass: $m / z 264$ (M-1) (calcd for $\mathrm{C}_{17} \mathrm{H}_{12}$ FNO: 265.09). FT-IR (KBr): $v_{\max }: 828(\mathrm{~m}), 1118(\mathrm{~m}), 1274(\mathrm{~m}), 3050(\mathrm{w}) \mathrm{cm}^{-1}$. Analytically calculated for $\mathrm{C}_{17} \mathrm{H}_{12}$ FNO: C 76.97, H 4.56, F 7.16, N, 5.28\%. Found: C 76.82; H 4.65; N 5.41\%.

$12 \mathrm{H}$-Benzo [5, 6] pyrrolizino [1, 2-b] quinoline (4a). Yellow needle crystal, mp: $128-130{ }^{\circ} \mathrm{C}$ (from MeOH). ${ }^{1} \mathrm{H}-\mathrm{NMR}(400$ $\left.\mathrm{MHz} \mathrm{CDCl}_{3}\right): \delta=5.28(\mathrm{~s}, 2 \mathrm{H}), 7.20(\mathrm{t}, J=4.0 \mathrm{~Hz}, 1 \mathrm{H}), 7.20(\mathrm{~s}$, $1 \mathrm{H}), 7.31(\mathrm{t}, J=7.6 \mathrm{~Hz}, 1 \mathrm{H}), 7.45(\mathrm{~d}, J=8.4 \mathrm{~Hz}, 1 \mathrm{H}), 7.55(\mathrm{t}, J=$ $4.0 \mathrm{~Hz}, 1 \mathrm{H}), 7.76(\mathrm{t}, J=8.0 \mathrm{~Hz}, 1 \mathrm{H}), 7.81(\mathrm{~d}, J=8.0 \mathrm{~Hz}, 1 \mathrm{H})$, 7.83 (d, $J=9.2 \mathrm{~Hz}, 1 \mathrm{H}), 8.16(\mathrm{~s}, 1 \mathrm{H}), 8.21$ (d, $J=8.8 \mathrm{~Hz}, 1 \mathrm{H})$ ppm. ${ }^{13} \mathrm{C}-\mathrm{NMR}\left(100 \mathrm{MHz}, \mathrm{CDCl}_{3}\right): \delta=46.5,95.3,109.5,120.4$, 122.8, 123.0, 126.3, 126.6, 127.9, 129.1, 129.8, 130.1, 132.7, 133.6, 134.4, 140.9, 148.5, 153.1 ppm. Mass: $\mathrm{m} / \mathrm{z} 256\left(\mathrm{M}^{+}\right)$ (calcd for $\mathrm{C}_{18} \mathrm{H}_{12} \mathrm{~N}_{2}$ : 256.10). FT-IR (KBr): $v_{\max }: 1318$ (m), 1570 (m), 2919 (w), 3003 (w) $\mathrm{cm}^{-1}$. Analytically calculated for $\mathrm{C}_{18} \mathrm{H}_{12} \mathrm{~N}_{2}$ : C 84.35, H 4.72, N 10.93\%. Found: C 84.42; H 4.61; N $10.65 \%$.

8-Chloro-12H-benzo [5, 6] pyrrolizino [1, 2-b] quinoline (4b). Yellow needle crystal, mp: $117-120{ }^{\circ} \mathrm{C}$ (from MeOH). ${ }^{1} \mathrm{H}-$ NMR (400 MHz, $\left.\mathrm{CDCl}_{3}\right): \delta=5.28(\mathrm{~s}, 2 \mathrm{H}), 7.12(\mathrm{~s}, 1 \mathrm{H}), 7.24(\mathrm{dd}$, $J=8.6 \mathrm{~Hz}, J=2.0 \mathrm{~Hz}, 1 \mathrm{H}), 7.36$ (d, $J=8.8 \mathrm{~Hz}, 1 \mathrm{H}), 7.58$ (t, $J=$ $7.6 \mathrm{~Hz}, 1 \mathrm{H}), 7.76-7.79(\mathrm{~m}, 2 \mathrm{H}), 7.84$ (d, $J=8.4 \mathrm{~Hz}, 1 \mathrm{H}), 8.17$ (s, $1 \mathrm{H}), 8.21$ (d, $J=8.4 \mathrm{~Hz}, 1 \mathrm{H})$ ppm. ${ }^{13} \mathrm{C}-\mathrm{NMR}\left(100 \mathrm{MHz}, \mathrm{CDCl}_{3}\right)$ : $\delta=46.7,110.5,122.0,123.4,126.2,126.6,127.9,130.0,130.3$, $132.8,133.4,133.5,149.3,150.8,153.3,153.9$ ppm. Mass: $\mathrm{m} / \mathrm{z}$ $290\left(\mathrm{M}^{+}\right.$) (calcd for $\mathrm{C}_{18} \mathrm{H}_{11} \mathrm{ClN}_{2}$ : 290.06). FT-IR (KBr): $v_{\text {max }}: 800$ (s), $1380(\mathrm{~m}), 1700(\mathrm{~m}), 2967(\mathrm{~m}) \mathrm{cm}^{-1}$. Analytically calculated for $\mathrm{C}_{18} \mathrm{H}_{11} \mathrm{ClN}$ : C 74.36, H 3.81, N 9.64\%. Found: C 74.23; H 3.70; $\mathrm{N}$ 9.53\%.

\section{Acknowledgments}

We are thankful to Alzahra University and Iran National Science Foundation (INSF) for financial support.

\section{References and notes}

1. (a) Yin, L.; Liebscher, J. Chem. Rev. 2007, 107, 133-137. (b) Alonso, F.; Beletskaya, I. P.; Yus, M. Tetrahedron 2005, 61, 1177111835. (c) Biffis, A.; Zecca, M.; Basato, M. J. Mol. Catal. A Chem. 2001, 173, 249-274.

2. (a) Jeffery, T. J. Chem. Soc., Chem. Commun. 1984, 1287-1289. (b) Bankston, D.; Fang, F.; Huie, E.; Xie, S. J. Org. Chem. 1999, 64, 34613466. (c) Jeffery, T. Synthesis 1987, 70-71. (d) Jeffery, T. Tetrahedron Lett. 1985, 26, 2667-2670.

3. (a) Beletskaya, I. P.; Cheprakov, A. V. Chem. Rev. 2000, 100, 30093066. (b) Dounay, A. B.; Overman, L. E. Chem. Rev. 2003, 103, 29452964. (c) Shibasaki, M.; Boden, C. D. J.; Kojima, A. Tetrahedron 1997, 53, 7371-7395.

4. Danishefsky, S. J.; Masters, J. J.; Young, W. B.; Link, J. T.; Snyder, L. B.; Magee, T. V.; Jung, D. K.; Isaacs, R. C. A.; Bornmann, W. G.; Alaimo, C. A.; Coburn, C. A.; Di Grandi, M. J. J. Am. Chem. Soc. 1996, 118, 2843-2859.

5. Abbas, S.; Ferris, L.; Norton, A. K.; Powell, L.; Robinson, G. E.; Siedlecki, P.; Southworth, R. J.; Stark, A.; Williams, E. G. Org. Process Res. Dev. 2008, 12, 202-212. 
6. Singh, J. B.; Bharadwaj, K. C.; Gupta, T.; Singh, R. M. RSC Adv. 2016, 6, 26993-26999.

7. (a) Li, W.; Gao, J. J.; Zhang, Y.; Tang, W.; Lee, H.; Fandrick, K. R.; Lu, B.; Senanayakea, C. H. Adv. Synth. Catal. 2011, 353, 1671-1675. (b) Kleeman, A.; Engel, J.; Kutscher, B.; Reichert, D. Pharmaceutical Substances. Synthesis, Patents, Applications, Georg Thieme Verlag, Stuttgart, Germany, 2001.

8. References therein: (a) Natarajan, J. K.; Alumasa, J. N.; Yearick, K.; Ekoue-Kovi, K. A.; Casabianca, L. B.; de Dios, A. C.; Wolf, C.; Roepe, P. D. J. Med. Chem. 2008, 51, 3466-3479. (b) De, D.; Krogstad, F. M.; Byers, L. D.; Krogstad, D. J. J. Med. Chem. 1998, 41, 4918-4926. (c) Delarue, S.; Girault, S.; Maes, L.; Debreu-Fontaine, M. A.; Labaeid, M.; Grellier, P.; Sergheraert, C. J. Med. Chem. 2001, 44, 2827-2833. (d) Stocks, P. A.; Raynes, K. J.; Bray, P. G.; Park, B. K.; O’Neill, P. M.; Ward, S. A. J. Med. Chem. 2002, 45, 4975-4983. (e) O'Neill, P. M.; Willock, D. J.; Hawley, S. R.; Bray, P. G.; Storr, R. C.; Ward, S. A.; Park, B. K. J. Med. Chem. 1997, 40, 437-448.

9. (a) Vu, A. T.; Campbell, A. N.; Harris, H. A.; Unwalla, R. J.; Manasc, E. S.; Mewshawa, R. E. Bioorg. Med. Chem. Lett. 2007, 40534056. (b) Vu, A. T.; Cohn, S. T.; Manas, E. S.; Harris, H. A.; Mewshaw, R. E. Bioorg. Med. Chem. Lett. 2005, 15, 4520-4525.

10. Hegab, M. I.; Abdel-Fattah, A. M.; Yousef, N. M.; Nour, H. F.; Mostafa, A. M.; Ellithey, M. Arch. Pharm. Chem. Life Sci. 2007, 340, 396-403.

11. (a) Sabitha, G.; Satheesh Babu, R.; Subba Reddy, B. V.; Yadav, J. S. Synth. Commun. 1999, 29, 4403-4408. (b) Rougeot, P. E.; Moskowitz, H.; Miocque, M. Tetrahedron Lett. 1983, 24; 2379-2382. (c) Ramesh, S.; Gaddam, V.; Nagarajan, R. Synlett 2010, 757-760. (d) Ibrahim, Y. A.; Moustafa, A. H. J. Chem. Res. (M) 1999, 4, 1231-1239 .

12. Yu, X.; Wang, J.; Xu, Z.; Yamamoto, Y.; Bao, M. Org. Lett. 2016 18, 2491-2494.

13. Aradi, K.; Bombicz, P.; Novk, Z. J. Org. Chem. 2016, 81, 920931.

14. (a) Faghihi, Z.; Oskooie, H. A.; Heravi, M. M.; Tajbakhsh, M.; Shiri, M. Monatsh Chem. 2016. (b) Shiri, M.; Faghihi, Z.; Oskouei, H. A.; Heravi, M. M.; Fazelzadeh, S.; Notashb, B. RSC Adv. 2016, 6, 9223592240. c) Shiri, M.; Zolfigol, M. A.; G. H.; Kruger, Tanbakouchian, Z. Friedländer annulation in the synthesis of azaheterocyclic compounds in the Advances in Heterocyclic Chemistry, A. R. Katritzky, Ed.; Elsevier Science, 2011, Vol. 102, pp. 139-227. d) Shiri, M.; Pourabed, R.; Zadsirjan, V.; Sodagar, E. Tetrahedron Lett. 2016, 57, 5435-5438. e) Shiri, M.; Zolfigol, M. A.; Pirveysian, M.; Ayazi-Nasrabadi, R.; Kruger, H. G.; Naicker, T.; Mohammadpoor-Baltork, I. Tetrahedron, 2012, 68, 6059-6064.

15. (a) Wang, G.-B.; Wang, L.-F.; Li, C.-Z.; Sun, J.; Zhou, G.-M.; Yang, D.-C. Res. Chem. Intermed. 2012, 38, 77-89. (b) Baruah, B.; Bhuyan, P. J. Tetrahedron 2009, 65, 7099-7104. (c) Kumar, S.; Bawa, S.; Drabu, S.; Panda, B. P. Med. Chem. Res. 2011, 20, 1340-1348. (d) Jawale, D. V.; Pratap, U. R.; Mane, R. A. Bull. Korean Chem. Soc. 2011, 32, 2171-2177. 


\section{Graphical Abstract}

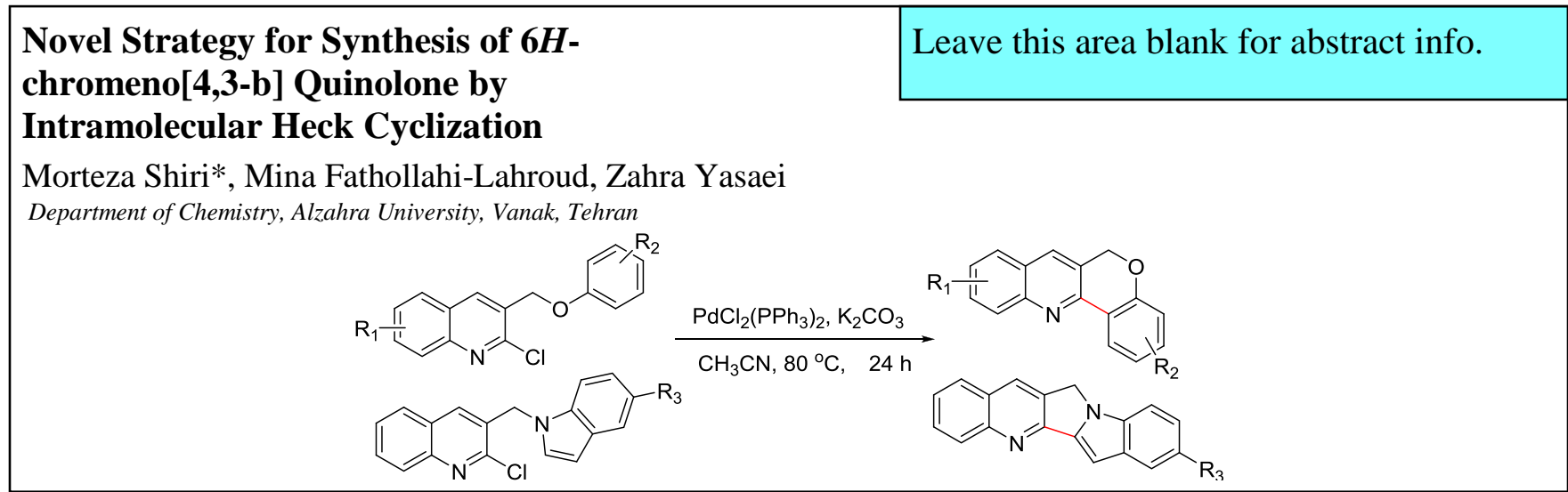

